\title{
Aufbau eines Vorgehensmodells zur Analyse von Haft-Gleit-Übergängen trockenlaufender Friktionspaarungen
}

\author{
Rüdiger Fehrenbacher ${ }^{1} \cdot$ Katharina Bause $^{1} \cdot$ Sascha Ott $^{1} \cdot$ Albert Albers $^{1}$ \\ Eingegangen: 17. Juni 2021 / Angenommen: 7. September 2021 / Online publiziert: 23. November 2021 \\ ○ Der/die Autor(en) 2021
}

\section{Zusammenfassung}

Die Auslegung des Kraftschlusses und der entsprechenden Haftreibung ist für die Funktionserfüllung trockenlaufender Kupplungen und Bremsen von grundlegender Bedeutung. Bei Systemen, in denen das Drehmoment möglichst ohne Relativbewegung der Reibpartner übertragen wird, ist das statische Reibmoment die maßgebende Auslegungsgröße. Grundlage bildet das Verständnis des Haft-Gleit-Übergangs, wobei dieser im System von vielen, situationsvariablen Größen wie Temperatur, Axialkraft und Drehmomentgradient sowie Eigenschaften wie der Belagart und Reibfläche beeinflusst wird. Den Schwerpunkt der Untersuchungen stellt die Sensitivitätsanalyse oben beschriebener Größen auf Basis von Messungen dar. Um die Einflüsse zu untersuchen, werden statische und quasistatische Versuchsverfahren unter Variation von Beanspruchungskollektiven anhand verschiedener Reibpaarungen unterschiedlicher Zustände analysiert. Hieraus ergeben sich Erkenntnisse hinsichtlich der verfahrensinduzierten sowie der tribosystemischen Einflüsse auf die Haft-Gleit-Übergänge der untersuchten Paarungen, welche für das Losbrechverhalten ausschlaggebend sind. Auf Basis der Untersuchungen werden Sensitivitäten in Bezug auf die Ermittlung des Losbrechens identifiziert und Methoden zur Messung und Auswertung des statischen Reibmoments abgeleitet, die unter den beschriebenen Voraussetzungen in Vorgehensmodelle integriert werden können.

\section{Development of a process model for the analysis of adhesion-slip transitions of dry-running friction couples}

\begin{abstract}
The dimensioning of the forced closure is of fundamental importance for the functional performance of dry-running clutches and brakes. The static friction torque determines the component's design and dimension for systems without any friction partners' relative movement. Understanding the adhesion-sliding transition is the basis for this parameter and is influenced by many variables and properties. Some of these are situation variables like Temperature, axial force, or torque gradient.

The main focus of the investigations is the sensitivity analysis of the above-described quantities based on measurements. To investigate the influences, static and quasi-static test procedures are analyzed under various stress collectives based on different friction pairings. The measures provide information about the tribology system's process-induced effects on the investigated pairings' adhesion-sliding transitions. Thus, sensitivities regarding the determination of the breakaway behavior are identified, and methods for measuring and evaluating the static frictional torque are generated, which can be integrated into process models under the described conditions.
\end{abstract}

\section{Einleitung}

Rüdiger Fehrenbacher

ruediger.fehrenbacher@kit.edu

1 IPEK - Institut für Produktentwicklung am Karlsruher Institut für Technologie (KIT),

Karlsruhe, Baden-Württemberg, Deutschland
Für trockenlaufende Kupplungen und Bremsen gibt es keine genormte Vorgehensweise zur Messung des statischen Reibmomentes [1-4]. Die Reibbelagshersteller nutzen i.d.R. sehr spezifische, hausinterne Prüfverfahren, um ihr Material zu charakterisieren. Der Komponentenherstel- 
ler hat wiederum ein davon abweichendes Messverfahren, um sein komplettes System zu testen. Letztlich wird diese Komponente im Gesamtsystem verbaut und muss dort die festgelegten Anforderungen erfüllen.

In der industriellen Praxis werden häufig die folgenden Messverfahren verwendet:

- Quasistatische Messungen bei konstanter Differenzdrehzahl (nahe Haften) sowie

- winkelabhängige Messung (Messung des Verdrehwinkels des geschlossenen Systems bei Einleitung eines Drehmomentes).

Beim zweiten Verfahren werden zwei Arten der Drehmomenteinleitung unterschieden:

- Aufbringen des Drehmoments mit kontinuierlichem Anstieg über der Zeit,

- Aufbringen des Drehmoments in Stufen (Stufen mit variabler Haltezeit).

Abhängig vom jeweilig gewählten Messverfahren werden teilweise stark abweichende Messergebnisse generiert. In der Diskussion mit Kunden und Komponentenlieferanten existiert nicht selten Unklarheit über die Art und Weise der Ermittlung der Messwerte. Abhilfe schafft hier nur eine Analyse und ein Vergleich der unterschiedlichen Messverfahren. Die notwendigen Vergleichsmessungen sind jedoch sehr zeit- und arbeitsintensiv. Zudem ist die Interpretation der Ergebnisse nur langjährigen Mitarbeitern mit großem Erfahrungswissen möglich.

Letztendlich besteht der Bedarf nach einer standardisierten Messmethode bzw. einem standardisierten Messverfahren unter identischen äußeren Einflüssen und gleichem Verhalten von Schnittstellen. Dieses Messverfahren soll eine geringe Streuung aufweisen, belastbare und reproduzierbare Messergebnisse generieren und robust gegenüber äußeren Störgrößen sein. Die Akzeptanz soll durch einen möglichst einfachen Aufbau sichergestellt werden, was vor allem für einige kleine und mittlere Unternehmen (KMU) eine notwendige Bedingung darstellt.

Die Autoren konzentrieren sich in den Untersuchungen vor allem auf die Form der Drehmomentaufbringung in Kombination mit unterschiedlichen Flächenpressungen und Temperaturen, um die daraus resultierenden Unterschiede des Losbrechverhaltens verschiedener Reibpaarungen zu analysieren und somit die beste Methode zur Bestimmung des statischen Reibmomentes zu erarbeiten.

Ziel ist aus den Untersuchungen des Friktionskontakts Erkenntnisse über den Haft-Gleit-Übergang und deren Einflussfaktoren zu gewinnen. Der zeitliche Verlauf der Reibungszahl während eines Versuchs in Verbindung mit den parallel aufgezeichneten Entwicklungen der Gleitgeschwindigkeit, des Verdrehwinkels und des Drehmomentes eröffnet neue Möglichkeiten bei der Beurteilung von Haft-GleitÜbergängen. Es hat sich gezeigt, dass die zeitlichen Verläufe dieser Messgrößen gegenseitiger, systemischer Wechselwirkungen unterliegen und von vielen weiteren (äußeren) Parametern abhängig sind, weshalb hier weitere Auswerteund Bewertungskriterien erarbeitet werden.

\section{Vorgehensmodell, Methoden und Auswertetools}

Abb. 1 stellt das erarbeitete Vorgehen dar und beschreibt die Methodenbausteine zur systematischen Untersuchung des statischen Reibmoments. Nachfolgend wird auf die einzelnen Bausteine eingegangen [5].

\subsection{Prüfstandsaufbau}

Die in Abb. 2 dargestellte Prüfumgebung bietet Möglichkeiten, die in der Industrie gebräuchlichen Messmethoden
Abb. 1 Vorgehensmodell und Methoden zur Ermittlung des statischen Reibmoments

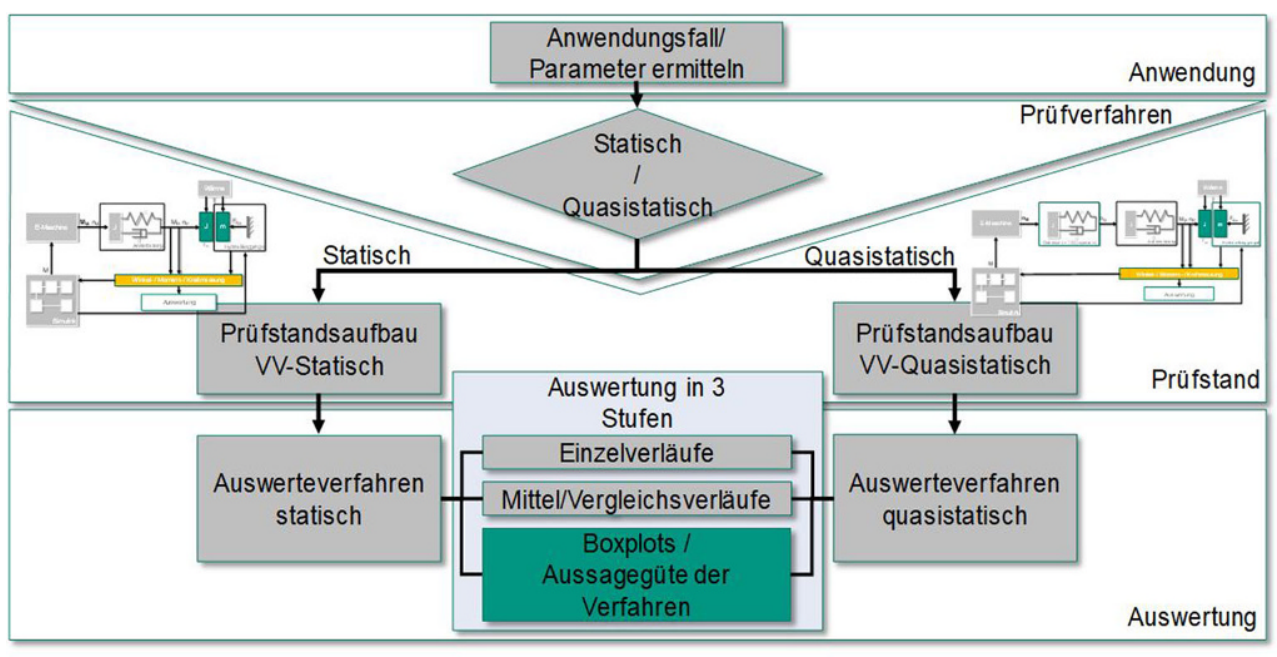




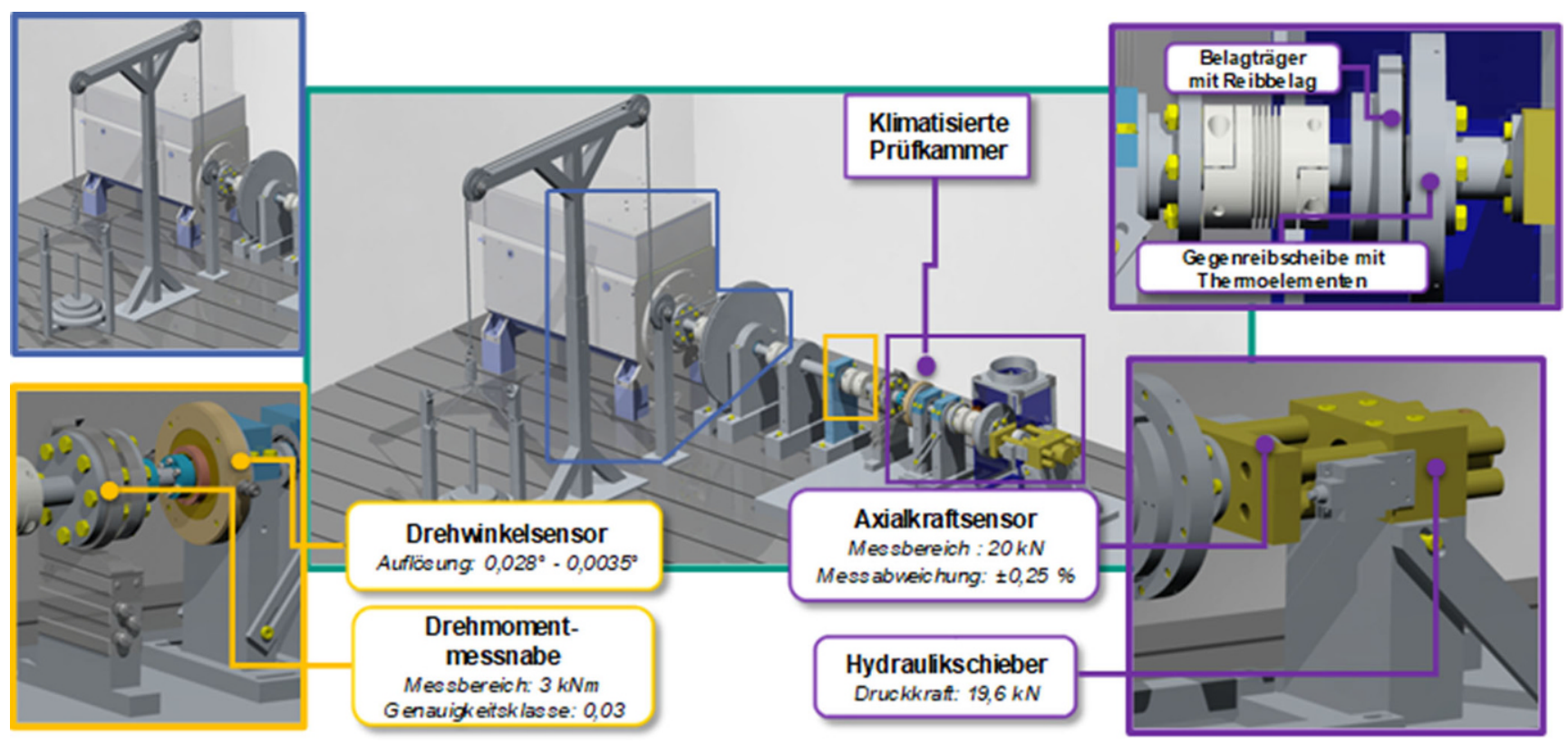

Abb. 2 Prüfinfrastruktur zur Ermittlung des statischen Reibmoments in unterschiedlichen Verfahren

gänzlich durchzuführen und bildet das zentrale Element zur Sicherstellung der Vergleichbarkeit auf physischer Ebene. Umgesetzt ist dies durch ein Umbaukonzept, das bei einem Verfahrenswechsel lediglich die Integration eines Maschinenelementes benötigt. Bei allen anderen Wechseln zwischen unterschiedlichen Prüfmethoden ist kein physisches Eingreifen erforderlich. Die Anforderungen an die auszuführenden Leistungen der Prüfinfrastruktur sind durch die Testmethoden der Industrie vorgegeben. Hierzu zählen die kontinuierliche und stufenartige Erhöhung des Drehmoments bis zum Losbrechen des verpressten Kontakts, das Aufprägen eines konstanten Minimalschlupfes über mehrere Minuten sowie die mehrere Tage andauernde Belastung des Friktionspaares mit einem konstanten Drehmoment nahe am zuvor ermittelten Losbrechmoment.

Der zentrale Aspekt ist die Integration des Reibkontakts. Daher ist die physische Modellierung des tribologischen Systems von besonderer Bedeutung. Um möglichst wenig Einflüsse des Prüfstandes auf die Messdaten zu haben, sollten die Messgrößen so nah wie möglich am Funktionskon- takt abgegriffen werden. Die konstruktive sowie softwaretechnische Umsetzung muss es ermöglichen, unterschiedliche Drehmomentcharakteristiken einzustellen und Relativbewegungen zwischen Reibbelag und Gegenreibscheibe zuzulassen.

Abb. 3 zeigt das auf Basis der Zielsetzung, Anforderungen und Randbedingungen erarbeitete Prinzip des Aufbaus zur Durchführung statischer Versuche. Der Prüfkopf umfasst die Anbindung der Reibbeläge und der Gegenreibscheibe mit direkt unter der Reibfläche angebrachten Temperatursensoren. Der Reibbelag ist an eine Synchronmaschine gekoppelt, die Gegenreibscheibe weist keinen rotatorischen Freiheitsgrad auf. Die Flächenpressung im Kontakt wird durch eine axiale Bewegung der gesamten Gegenreibscheibe über einen hydraulischen Zylinder aufgebracht und das Drehmoment über den Rotationsfreiheitsgrad des Reibbelags in den Friktionskontakt eingeleitet [6]. Der Messbereich des Axialkraftsensor liegt bei $20 \mathrm{kN}$, die Maximalkraft in dieser Versuchsreihe beträgt $7 \mathrm{kN}$ was einer Abweichung von max. $\pm 17,5 \mathrm{~N}$ entspricht.
Abb. 3 Prinzipskizze des statischen Vorgehens [6]

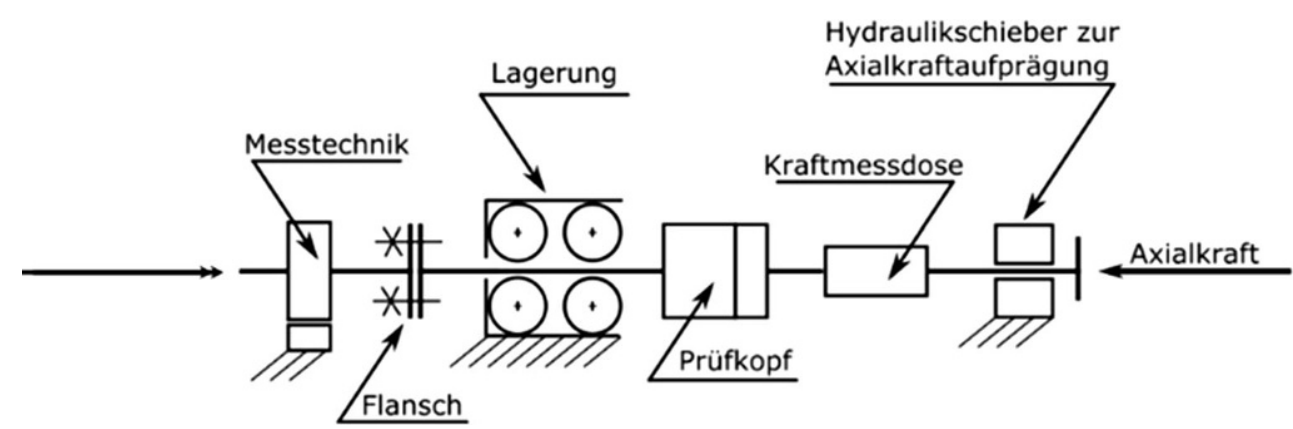




\subsection{Relevante Größen}

Der zeitliche Verlauf der Reibungszahl während eines Versuchs erlaubt Rückschlüsse auf das Verhalten der Reibpaarung während der Haft-Gleit-Übergänge. Bei der Reibungszahl handelt es sich um eine berechnete Größe, welche wiederum aus errechneten Werten wie dem mittleren Reibradius der Friktionspaarung sowie dem aktuell gemessenen Drehmoment ermittelt wird. So wird die Reibungszahl und vor allem deren zeitlichen Verlauf u. a. von der Flächenpressung, dem Zustand des Belags (Topographie, Belastungshistorie, ...), Feuchtigkeit, Temperatur aber auch durch die Eigenschaften des Restsystems und v.a. der Systemreaktion beeinflusst $[7,8]$. Wie zuvor beschrieben genügt die Angabe eines Wertes für das statische Reibmoment nicht, um die komplexen Vorgänge im Haft-Gleit-Verhalten zu beschreiben. Neben dem Drehmomenten- und dem Reibungszahlverlauf über der Zeit werden somit weitere Größen und deren Korrelationen zur Analyse des Losbrechverhaltens herangezogen. Die dafür zu messenden Systemgrößen sind Axialkraft sowie der Abstand der beiden Reibflächen zueinander, Drehmoment und der Relativwinkel sowie die Temperatur, welche permanent über $3 \times 4$ Messtellen direkt unter der Reibfläche der Gegenreibscheibe aufgezeichnet wird. Die berechneten Größen sind Gleitgeschwindigkeit, Reibungszahl und Flächenpressung.

\subsection{Beschreibung des statischen Versuchsverfahrens}

Im statischen Versuchsverfahren wird der Aufbau gemäß Abb. 2 und 3 verwendet. Gemäß dem Versuchsablauf (Abb. 4) werden zwei Reibpaarungen untersucht. Hierbei wird das Material (C45) der Gegenreibscheibe beibehalten und mit einem organischen, ringförmigen, massen- gepressten sowie mit einem metallischen, segmentierten, gesinterten Belag gepaart.

Um auch die Richtungsabhängigkeit des Haft-GleitÜberganges zu untersuchen, werden in jedem Betriebspunkt 19 Schaltungen im Linkslauf gefahren sowie eine Schaltung im Rechtslauf. Die Axialkraft wird aufgebaut und verpresst die Gegenreibscheibe mit dem Reibbelag. Hat die Axialkraft den zuvor definierten Wert erreicht, wird an der E-Maschine mit der im Steuer- und Regelmodell hinterlegten Dynamik solange eine Drehmomentsteigerung bewirkt, bis der Reibkontakt losbricht. Der Versuchslauf ist beendet, wenn eine zuvor definierte Gleitgeschwindigkeit von $1,3 \mathrm{~mm} / \mathrm{s}$ detektiert wird (Abb. 4, Kreis). Abb. 5 zeigt eine Untersuchung unterschiedlicher Abbruchkriterien. Hierbei wird deutlich, dass die Verwendung des Winkels als Abbruchkriterium zu geringeren berechneten Reibungszahlen führt, jedoch mit einer höheren Streuung einhergeht. Letzteres begründet sich in der Anfälligkeit, Messrauschen als Bewegung fehlzuinterpretieren. Im Anschluss wird die Axialkraft abgebaut und der Reibbelag stochastisch lastfrei relativ zur Gegenreibscheibe verdreht. Damit wird sichergestellt, dass bei der nächsten Schaltung nicht lokal dieselben Reibkontakte aufeinandertreffen und den Funktionskontakt bilden.

\subsection{Beschreibung des quasistatischen Versuchsverfahrens}

Abb. 6 zeigt den schematischen Aufbau, der für die Versuche im quasistatischen Versuchsverfahren genutzt wird. Zur Aufprägung der Gleitgeschwindigkeit wird die E-Maschine drehzahlgesteuert betrieben. Um die zu untersuchenden, sehr niedrigen Gleitgeschwindigkeiten darstellen zu können, wird ein spielfreies Getriebe mit der Untersetzung von 1:100 eingesetzt. Dieses wird, ohne weitere Änderun-

Abb. 4 Schematischer zeitlicher Verlauf der Eingangsgrößen im statischen Verfahren

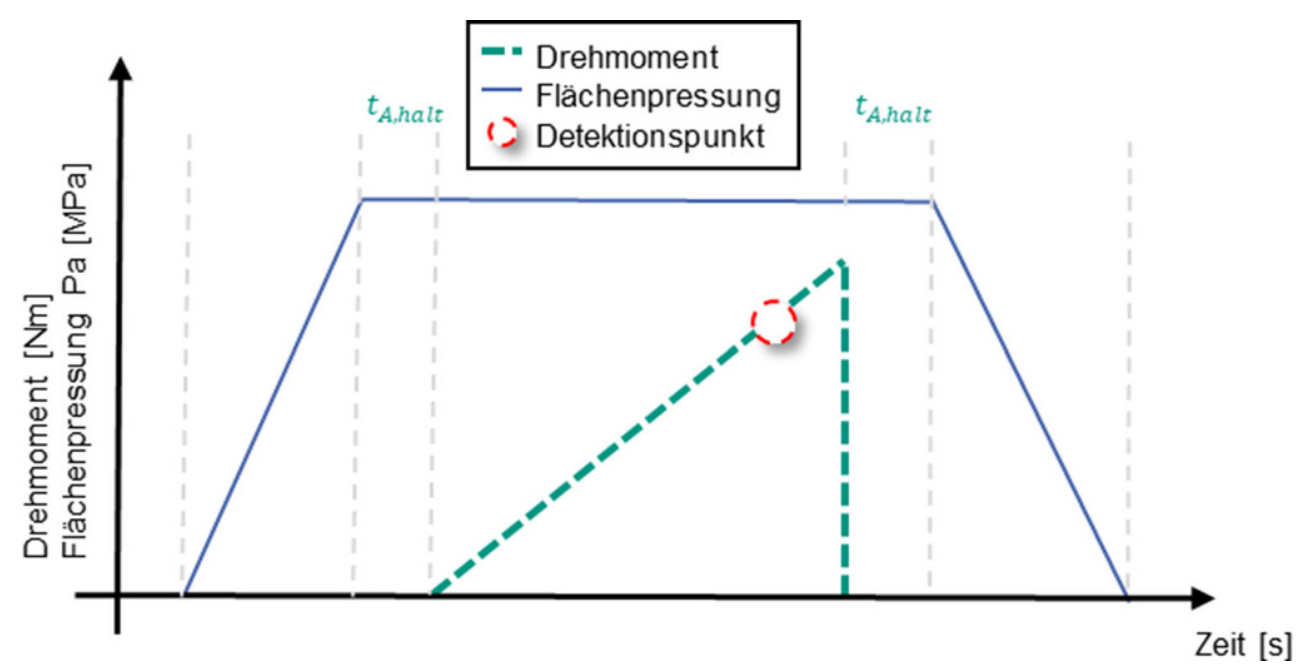



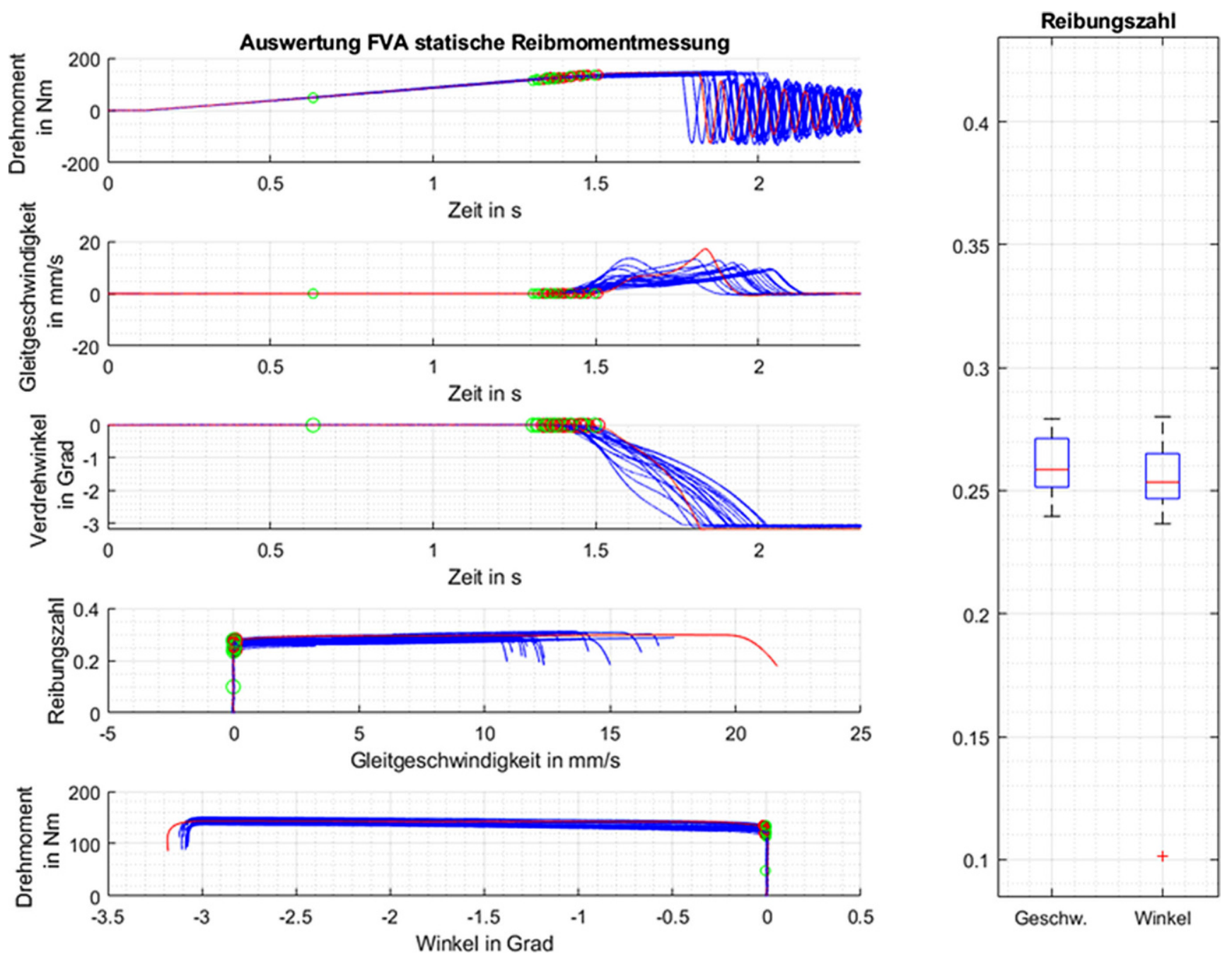

Abb. 5 Vergleich der Abbruchkriterien

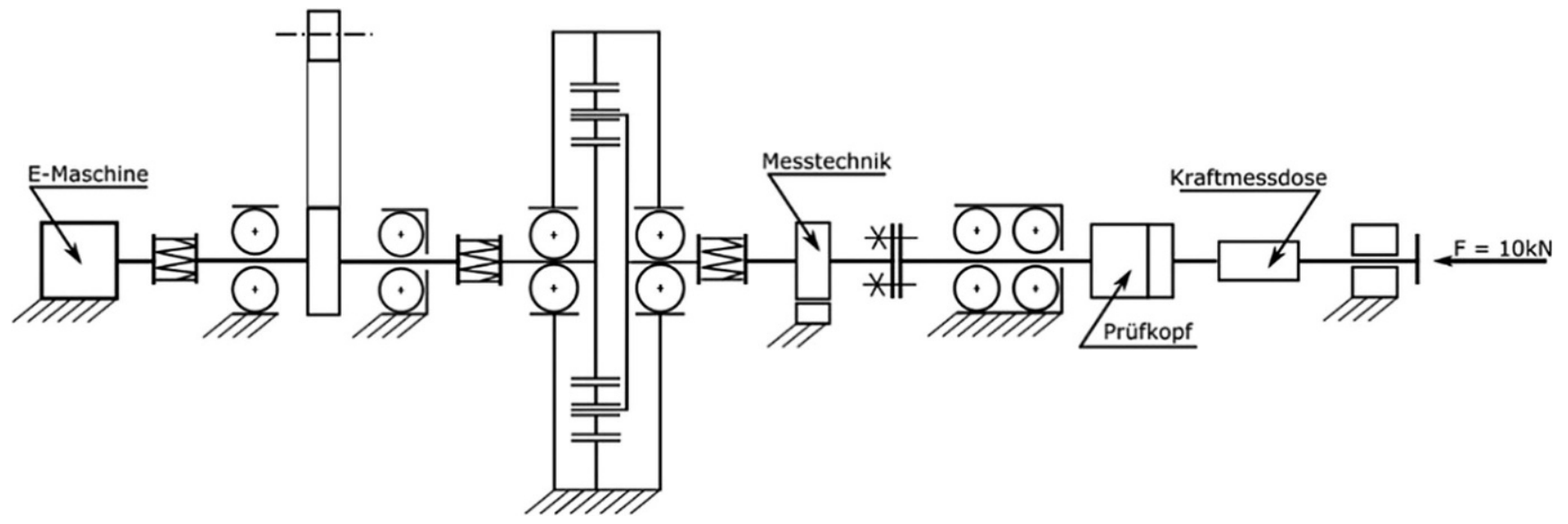

Abb. 6 Prinzipskizze des quasistatischen Verfahrens [6] 


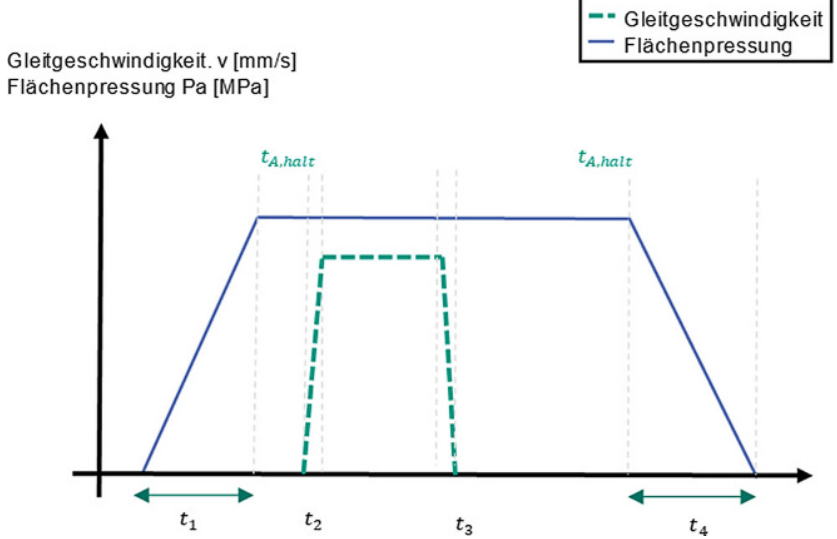

Abb. 7 Schematischer, zeitlicher Verlauf der Eingangsgrößen im quasistatischen Verfahren

gen des Restsystems, anstelle der Überbrückungswelle (vgl. Abb. 2 und 3) integriert.

Der Ablauf einer quasistatischen Schaltung (Abb. 7) ist wie folgt umgesetzt: Der Aufbau der Flächenpressung entspricht dem des statischen Verfahrens. Nach Erreichen der geplanten Axialkraft wird an der E-Maschine die Drehzahl und somit die dem Kontakt aufzuprägende Gleitgeschwindigkeit gestellt. Sobald sich diese stabilisiert hat, wird der Wert über $5 \mathrm{~s}$ gehalten.

\subsection{Beschreibung der Stichversuche mit reduziertem Parameterraum}

In einem ersten Stichversuch werden die Verfahren statisch und quasistatisch mit reduziertem Parameterumfang bei drei höheren Temperaturen von 40,60 und $70^{\circ} \mathrm{C}$ wiederholt. Die Steuerung der Eingangsparameter ist identisch zu den Grundverfahren. In einem zweiten Stichversuch wird das Drehmoment nicht kontinuierlich, sondern sprunghaft, stufenförmig in einem Abstand von mehreren Sekunden erhöht. In einem dritten Stichversuch wird jedem Drehmomentsprung (Stichversuch II) eine zusätzliche Drehmo- mentschwingung überlagert. Das Halten der Eingangsgrößen über mehrere Sekunden schafft die Möglichkeit der genaueren Haft-Gleit-Übergangsbeurteilung. Eine derartige Anregung kann entweder zum Losbrechen mit anschlieBender Stabilisierung (Haften) oder zur Weiterbewegung (Gleiten) des Systems führen. Gleichsam dienen die Ergebnisse der beiden letzteren Versuche dem Abgleich mit den Ergebnissen aus den Stichversuchen IV. Hierdurch soll gezeigt werden, dass es möglich ist, den Einfluss einer stufenartigen Drehmomenterhöhung mit überlagerter Schwingung auf das Verhalten des Funktionskontakts ohne zusätzliche Einflüsse des Versuchsdurchführenden (vgl. Stichversuch IV) automatisiert nachzubilden. Der vierte Stichversuch bildet eines der gängigen Verfahren in der industriellen Praxis ab. Über Massen respektive Hebel wird ebenfalls eine stufenartige Beanspruchungserhöhung des Kontakts bis zum Losbrechen erwirkt. Diese Prüfung dient dazu, das Zeitverhalten einer auf Dauer geschlossenen Sicherheitsbremse abzubilden. Indem der Kontakt mit einem Drehmoment nahe an der zuvor ermittelten Rutschgrenze über Stunden respektive Tage beaufschlagt wird, um so das Kriechen eines Funktionskontakts über der Zeit festzustellen. Hierbei wird beobachtet, dass in dem Kontakt eine sehr geringe Relativbewegung stattfindet. Das hierfür verwendete Modul ist in Abb. 2 (blauer Bereich) dargestellt.

\subsection{Auswertemethoden}

Die Analyse der Messdaten erfolgt über drei erarbeitete Auswertungsstufen (Abb. 8), deren Ergebnisdarstellung verfahrensübergreifend vergleichbar ist. Ebene 1 schafft hierbei den Vergleich zwischen den einzelnen Schaltungen eines Betriebspunktes des Versuchsplan. Ebene 2 fasst die Schaltungen pro Betriebspunkt für die einzelnen Messgrößen zu einem Median zusammen und ermöglicht so die Einflussanalyse der variierten Parameter über mehrere Messungen hinweg, indem über diese gefiltert wird. Ebene 3 ermöglicht mittels Boxplots eine unkomplizierte Beurtei-
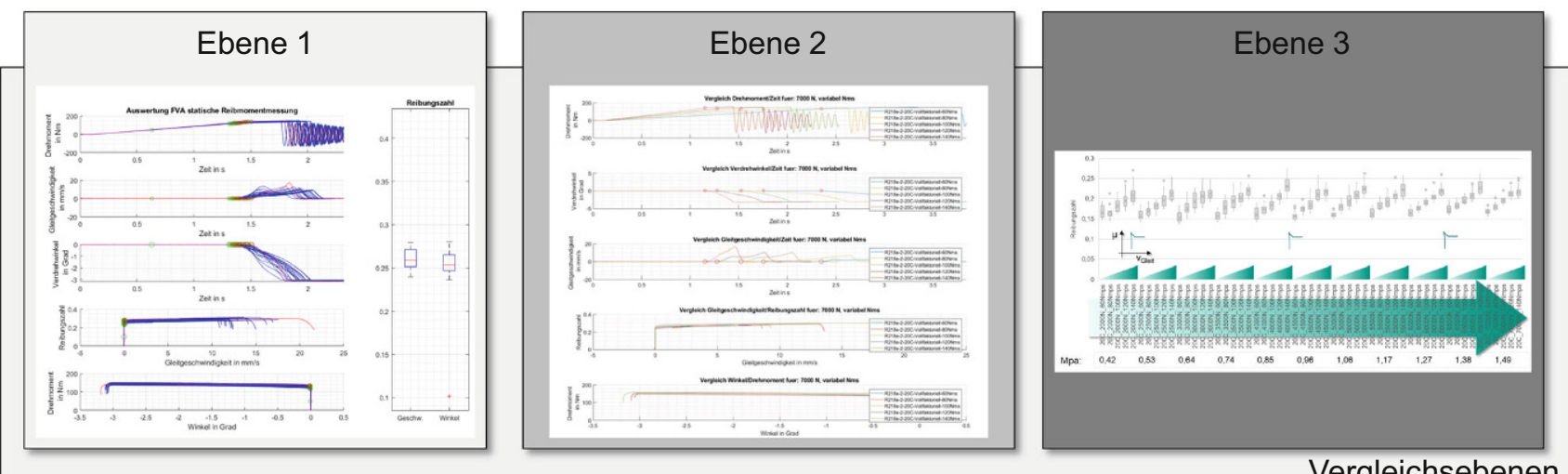

Vergleichsebenen

Abb. 8 Exemplarische Darstellung der Auswertung 
lung der Streuung, der Mittelwerte und Trends innerhalb der Verfahren aber auch über die Verfahren hinweg. Die Visualisierung kann über wenige Handgriffe per GUI erstellt werden. Hierzu greift eine eigens implementierte Software auf die hinterlegten Messdaten $\mathrm{zu}$ und operationalisiert Auswertung und Interpretation der Messdaten.

\section{Ergebnisse}

\subsection{Statisches Verfahren}

Da in den zukünftigen Anwendungen die Systemeigenschaften wie Bauraum oder auch Axialkraft festgelegt sind, liegt der Fokus der zu beurteilenden Größen vor allem auf den Rampen des Drehmoments und der Temperatur. Der organische Belag zeigt in neuem Zustand über alle gefahrenen Drehmomentrampen hinweg ein gleiches Losbrechverhalten (Abb. 9a). Die Zunahme der Reibungszahl mit Erhöhung der Drehmomentrampe zeigt sich ebenfalls bei den Versuchen mit Belägen, welche eine Belastungshistorie aufweisen.

Die Verläufe der Paarung mit metallischem Belag (Abb. 9b) zeigen ein für die spätere Anwendung wünschenswertes Verhalten. Der Haft-Gleit-Übergang unterscheidet sich vom organischen Belag durch einen weiteren, leichten Anstieg der Reibungszahl unmittelbar nach dem Losbrechen. Durch dieses Verhalten wird ein starker Drehzahlsprung unterbunden.
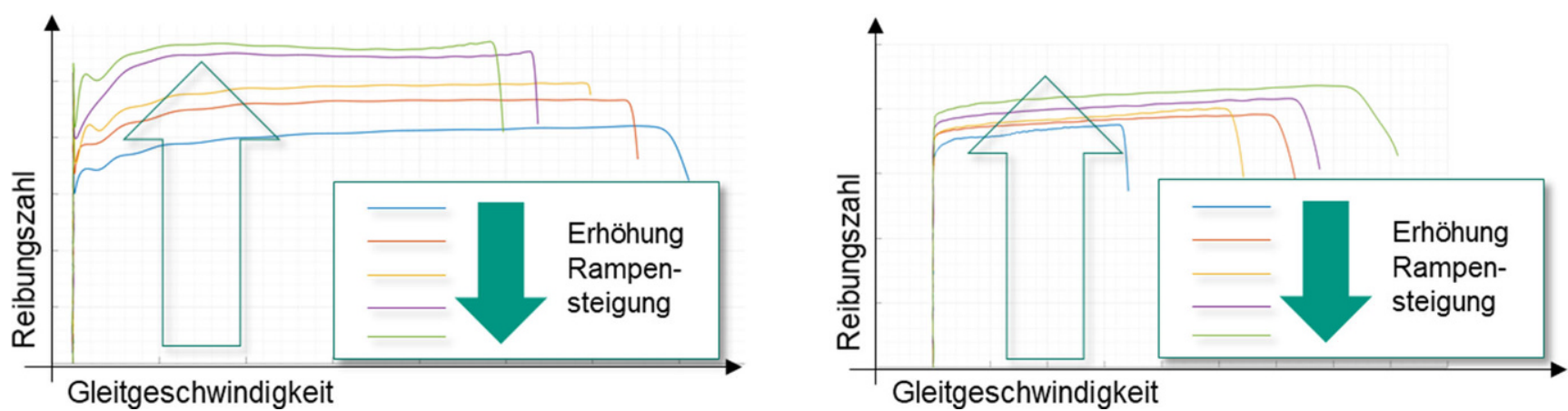

Abb. 9 Reibungszahlverläufe bei steigenden Drehmomentrampen (a organischer; b metallischer Belag)
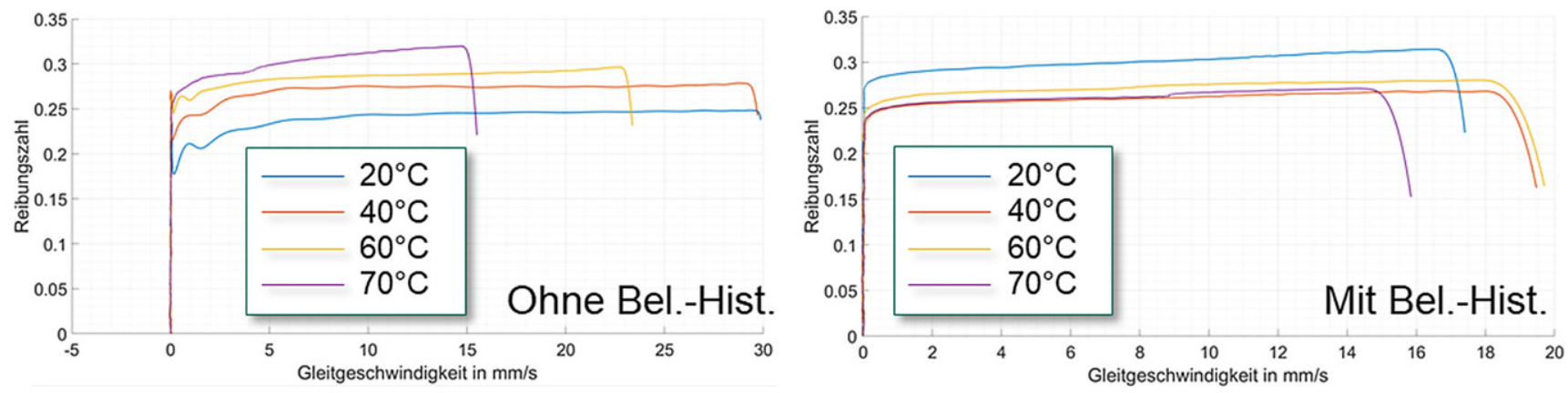

Abb. 10 Reibungszahlverlauf bei verschiedenen Temperaturen des organischen Belages (a ohne; b mit Belastungshistorie)
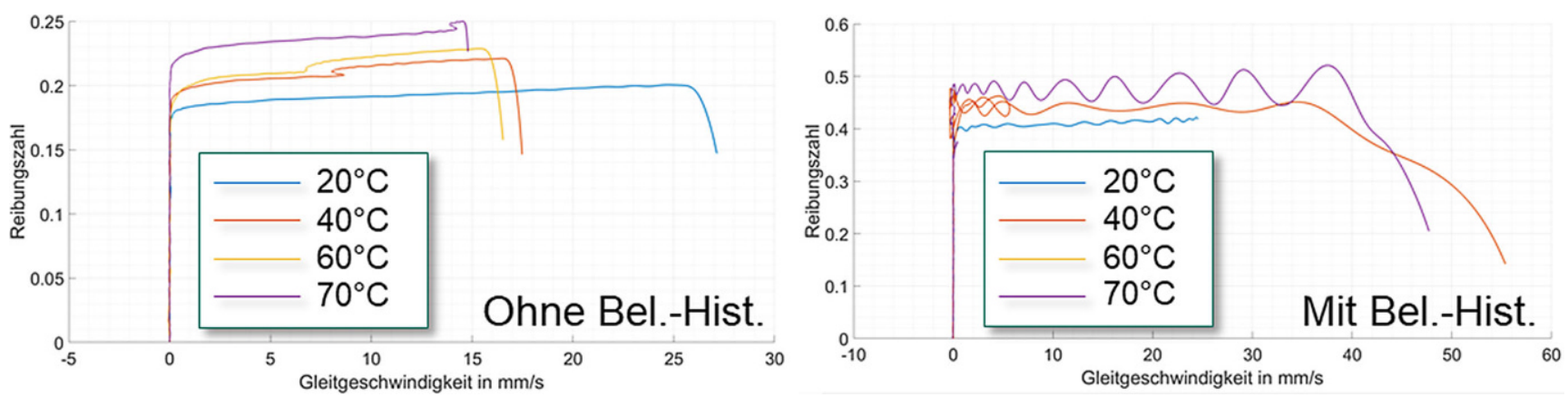

Abb. 11 Reibungszahlverlauf bei verschiedenen Temperaturen des metallischen Belages (a ohne; b mit Belastungshistorie) 
Ein anderes Verhalten zeigt sich bei der Auswertung der Versuche mit Erhöhung der Temperatur. Betrachtet man zunächst nur den Einfluss der ins System eingebrachten Wärmemenge, steigt die mittlere Reibungszahl beim organischen Belag ohne Belastungshistorie an. (Abb. 10a) Weiter zeigt sich bei allen Temperaturstufen ein starker Abfall der Reibungszahl unmittelbar nach dem Losbrechen. Interessant ist das Verhalten des Belages nach einem verkürzten Einlauf. (Abb. 10b) Der hier beobachtete Haft-Gleit-Übergang ist geprägt durch eine weitere leichte Zunahme des Drehmomentes unmittelbar nach dem Losbrechen. Es gibt zwischen dem organischen und den metallischen Belag Parallelen bei einer Temperaturerhöhung. In neuem Zustand steigt die Reibungszahl mit der Temperaturerhöhung an, jedoch ist ein abweichendes Losbrechverhalten zu erkennen.

Der Reibungszahlverlauf der metallischen Paarung ohne Belastungshistorie gleicht dem des organischen Belages mit Belastungshistorie und steigt auch unmittelbar nach dem Losbrechen leicht an. (Abb. 11a) Die Verläufe mit Belastungshistorie weisen jedoch einen starken Rückgang während des Übergangs vom Haften ins Gleiten auf. (Abb. 11b) Der Einfluss der Temperaturerhöhung ist bei dieser Reibmaterialkombination weniger deutlich als bei der Paarung ohne Belastungshistorie. Jedoch treten vor allem bei $70^{\circ} \mathrm{C}$ durch die hohen Reibungszahlen beim Belag mit Belastungshistorie Wechselwirkungen mit dem Testsystem (hier: Prüfumgebung) auf, die zu ausgeprägteren Schwingungen führen.

Die maßgebenden Parameter, welche Sensitivitäten bezüglich Streuung und Reibungszahlniveau begünstigen, sind im statischen Verfahren die oftmals durch die Anwendung vorgegebenen Parameter Axialkraft sowie Temperatur. Messungen bei hohen Axialkräften sind tendenziell weniger streuungsbehaftet.

\subsection{Quasistatisches Verfahren}

Bei dem quasistatischen Verfahren werden vorrangig die Eingangsparameter, Drehzahl und Flächenpressung respektive Axialkraft variiert. Auffällig hier ist die mit Drehzahlerhöhung einhergehende Frequenzsteigerung des Drehmomentverlaufes beim organischen Belag. (Abb. 12) Eine Veränderung des mittleren Drehmoment- und somit Reibungszahlverlaufes ist nicht beobachtbar.

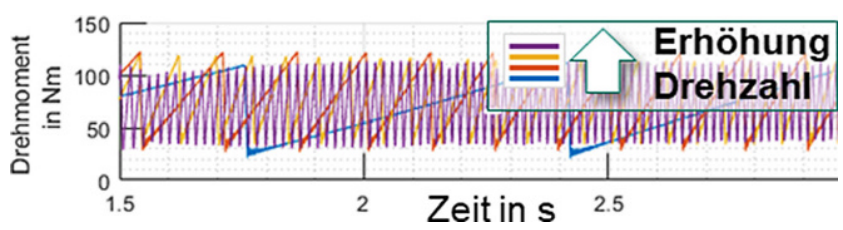

Abb. 12 Zeitlicher Drehmomentverlauf eines organischen Belages

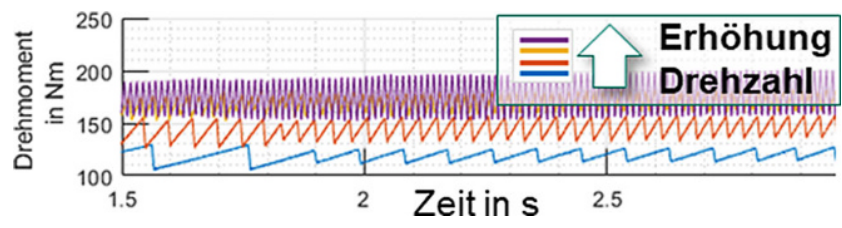

Abb. 13 Zeitlicher Drehmomentverlauf eines metallischen Belages

Ein anderes Verhalten lässt sich beim metallischen Reibbelagmaterial feststellen. Hier ist ersichtlich, dass eine Erhöhung der Drehzahl eine Erhöhung des resultierenden Drehmomentes bewirkt. (Abb. 13) Der Frequenzbereich unterliegt jedoch einer geringeren Spreizung. Die Werte der Amplituden steigen mit höheren Drehzahlen geringfügig.

Im stabilen Gleitbereich stellen sich teils starke Amplitudenausschläge ein, die um ein sich ausbildendes mittleres Drehmoment schwingen. Bestimmt wird dieses durch die eingestellten Größen Axialkraft und Gleitgeschwindigkeit. Die Gleitgeschwindigkeit beeinflusst dabei die Frequenz der Schwingung. Die Amplitude resultiert aus der Wechselwirkung der Belagseigenschaften (Belagszustand, -geometrie, -steifigkeit) mit den Steifigkeiten des Restsystems (hier Prüfstand). [9, 10].

\section{Diskussion}

Die verfahrensimmanente Herausforderung besteht in der Detektion und Beschreibung des Haft-Gleit-Übergangs. Die in diesem Vorhaben entwickelten Methoden und Vorgehensmodelle versuchen dem Rechnung zu tragen. Die durchgeführte Analyse der Charakteristik zeigt, dass neben den Belagseigenschaften auch das Beanspruchungskollektiv sowie der Belagszustand einen Einfluss darauf nimmt, ob die Haftreibungszahl im Losbrechen exponentiell abfällt, sich nach dem linearen Anstieg direkt auf Gleitreibungsniveau stabilisiert oder im weiteren Verlauf leicht steigt. Hieraus wird die Schlussfolgerung abgeleitet, dass eine Festlegung

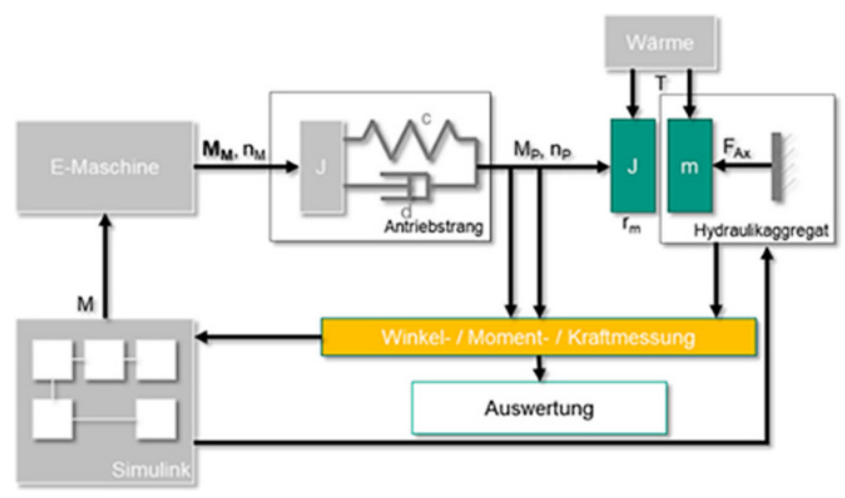

Abb. 14 Systembild der Prüfinfrastruktur im statischen Vorgehen 
Abb. 15 Vergleich sich ausbauender Schwingungen durch Reduzierung der Drehmomentrampen

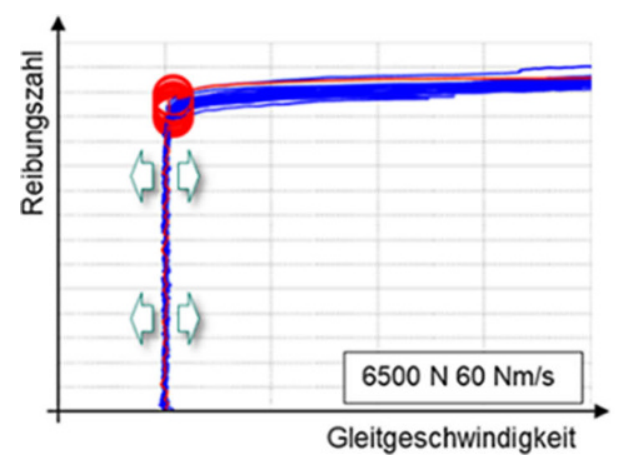

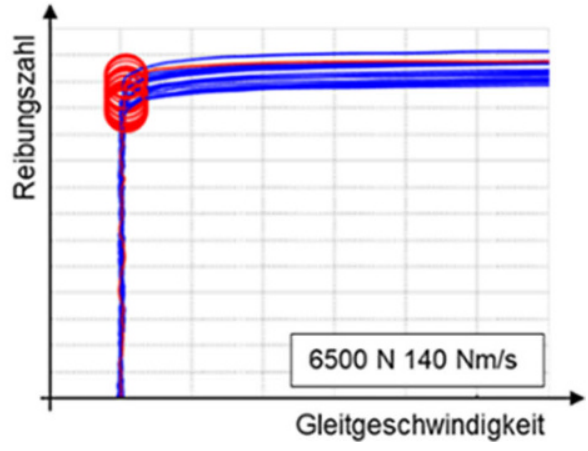
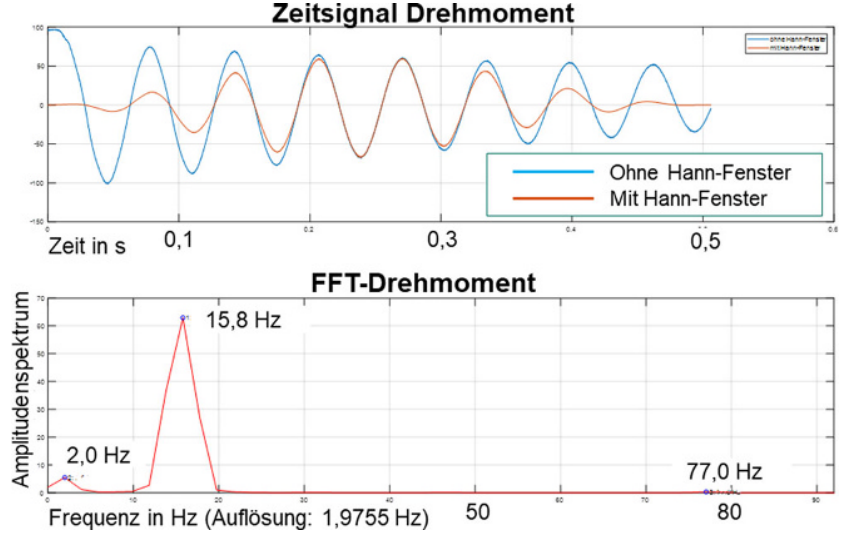

Abb. 16 FFT-Analyseergebnisse der Prüfstandsschwingung

des statischen Moments auf das maximal auftretende Moment nur teilweise zutrifft.

Eine Einflussnahme bezüglich des Zeitpunktes des Losbrechens kann auch durch Abbildung des Systembilds veranschaulicht werden. (Abb. 14) Wechselwirkungen mit Eigenschaften des Restsystem wie Steifigkeit oder Trägheit können zum Beispiel zu höheren Schwingungsamplituden bei geringerer Rampensteigung führen. (Abb. 15).

Darüber hinaus nimmt die Prüfstandsintegration des Reibkontakts beim statischen Verfahren einen großen Einfluss. Dies zeigt sich insbesondere bei Versuchen mit einem metallischen, segmentiert angebrachten Belag. Neben der Form des Belages, seiner Oberflächentopografie sowie dessen Steifigkeit (Abb. 14) wirken sich darüber hinaus etwaige Schiefstellungen oder Achsfehler aus, die dazu führen, dass der tatsächliche Wert des Reibradius vom theoretischen abweicht und während des Haft-Gleit-Übergang sowie über die Schaltungen und Belastungshistorie schwankt.

Ein weiterer Einflussfaktor auf die Streuung ist der Belagszustand, d.h. die während des Betriebes entstandene respektive gezielt hergestellte reibaktive Schicht, die nach Musiol [11] die bestimmende Größe der Eigenschaften einer Reibpaarung darstellt. Vor allem bei den Versuchen mit dem metallischen Belag zeigt sich eine Verbreiterung des Streubandes nach verkürzten Vorkonditionierungspro- zessen. Auch im quasistatischen Verfahren wirken sich anwendungsspezifische Parameter auf die Aussagegüte des Verfahrens aus, wobei insbesondere der Einfluss der Axialkraft sich nicht bzw. nur geringfügig auf die Streuung auszuwirken scheint. Die maßgebende Steuergröße des quasistatischen Verfahrens (Gleitgeschwindigkeit) scheint in Wechselwirkung mit dem Friktionskontakt, dessen Eigenschaften und dem Restsystem der Haupteinfluss auf die ermittelten Reibungszahlen und deren Streuung zu sein. Die Wechselwirkung zwischen dem Friktionskontakt und dem Restsystem existiert sowohl in der Anwendung als auch in der Prüfstandsintegration, sodass darauf zu achten ist, die Steifigkeit und auch die Dämpfungseigenschaften des Prüfaufbaus nahe dem der zukünftigen Anwendung zu wählen. Eigenfrequenzanalysen, wie die in diesem Projekt herangezogene (FFT-Analyse, Fast-Fourier-Transformation) (Abb. 16) können hierbei gute Anhaltspunkte liefern und zukünftig in der Entwicklung unterstützen.

Des Weiteren wurden hochdynamische PMSM (Permanentmagnet-Synchronmotoren) mit einer maximalen Anregungsfrequenz von $500 \mathrm{~Hz}$ als Antriebsmaschinen verwendet. Diese besitzen eine maximale Leistung von $300 \mathrm{~kW}$ bei einer Nenndrehzahl von 1000 1/min. Basierend auf einer Rotorträgheit von $0,75 \mathrm{kgm}^{2}$ können in der Kombination mit dem verwendeten Echtzeitsystem Beschleunigungen von bis zu $3800 \mathrm{rad} / \mathrm{s}^{\wedge} 2$ abgebildet werden.

Es ist zu verifizieren ob auch Standard E-Maschinen in der Lage sind, Drehmomentrampen dieser Präzision aufzubauen. Gerade im Bezug zu den Stufenversuchen konnte keine Verifizierung einer Standardantriebsmaschine unternommen werden. Die in diesem Projekt untersuchten Beläge mit ihren Eigenschaften unterscheiden sich bezüglich des Losbrechverhaltens teilweise deutlich voneinander. Daher ist es empfehlenswert zusätzlich andere Belagsmaterialien und Bauformen zu untersuchen.

Funding Open Access funding enabled and organized by Projekt DEAL.

Interessenkonflikt R. Fehrenbacher, K. Bause, S. Ott und A. Albers geben an, dass kein Interessenkonflikt besteht. 
Open Access Dieser Artikel wird unter der Creative Commons Namensnennung 4.0 International Lizenz veröffentlicht, welche die Nutzung, Vervielfältigung, Bearbeitung, Verbreitung und Wiedergabe in jeglichem Medium und Format erlaubt, sofern Sie den/die ursprünglichen Autor(en) und die Quelle ordnungsgemäß nennen, einen Link zur Creative Commons Lizenz beifügen und angeben, ob Änderungen vorgenommen wurden.

Die in diesem Artikel enthaltenen Bilder und sonstiges Drittmaterial unterliegen ebenfalls der genannten Creative Commons Lizenz, sofern sich aus der Abbildungslegende nichts anderes ergibt. Sofern das betreffende Material nicht unter der genannten Creative Commons Lizenz steht und die betreffende Handlung nicht nach gesetzlichen Vorschriften erlaubt ist, ist für die oben aufgeführten Weiterverwendungen des Materials die Einwilligung des jeweiligen Rechteinhabers einzuholen.

Weitere Details zur Lizenz entnehmen Sie bitte der Lizenzinformation auf http://creativecommons.org/licenses/by/4.0/deed.de.

\section{Literatur}

1. Richtlinie VDI 2241 (1982) Blatt 1 - Schaltbare fremdbetätigte Reibkupplungen und -bremsen

2. Richtlinie VDI 2241 (1982) Blatt 2 - Schaltbare fremdbetätigte Reibkupplungen und -bremsen

3. 0580:2011-11. VDE 0580: Elektromagnetische Geräte und Komponenten - Allgemeine Bestimmungen.

4. 2010-06. DIN EN 81-1: Sicherheitsregeln für die Konstruktion und den Einbau von Aufzügen - Teil 1: Elektrisch betriebene Personenund Lastenaufzüge

5. Fehrenbacher R, Ott S, Bause K (2019) Entwicklung einer Prüfmethode zur Ermittlung des statischen Reibmomentes (Losreißmo- ment) trockenlaufender Kupplungen und Bremsen auf Komponentenebene. Gesellschaft für Tribolgie (GfT), Göttingen

6. Mandel C, Wolter K, Bause K, Behrendt M, Hanf MM, Albers A (2020) Model-Based Systems Engineering methods to support the reuse of knowledge within the development of validation environments. In: I E E E (Hrsg) SysCon 2020. 14th Annual IEEE International Systems Conference

7. Kniel J (2017) Potenziale der Temperaturverteilungsmessung zur Analyse trockenlaufender Friktionssysteme auf Basis neuer Messtechnik. Karlsruher Institut für Technologie, Karlsruhe

8. Behrendt M (2009) Entwicklung eines Systemtribometers mit Abbildung mehrachsiger instationärer Beanspruchungskollektive zur Analyse von Reibung und Verschleiß im Mischreibungsgebiet im Kontext nasslaufender Umschlingungs-CVT. Universität Karlsruhe (TH), Karlsruhe

9. Fehrenbacher R, Ott S, Bause K (2019) Statische Reibmomentmessung. Entwicklung einer Prüfmethode zur Ermittlung des statischen Reibmomentes (Losreißmoment) trockenlaufender Kupplungen und Bremsen auf Komponentenebene. Abschlussbericht FVANr. 806 I. FVA-Heft, Bd. 1373. FVA Forschungsvereinigung Antriebstechnik e. V., Frankfurt a. M.

10. Fehrenbacher R, Ott S, Albers A (2019) Entwicklung einer Prüfmethode zur Ermittlung des statischen Reibmomentes (Losreißmoment) trockenlaufender Kupplungen und Bremsen auf Komponentenebene. In: Tagungsband FVA Informationstagung

11. Musiol F (1994) Erklärung der Vorgänge in der Kontaktzone von trockenlaufenden Reibpaarungen über gesetzmäßig auftretende Phänomene im Reibprozess. Dissertation TU-Berlin, Berlin 